\title{
The Rise of Particle Beam Therapy: Are We Ready for The Potential Game-Changer?
}

\author{
Eui Kyu Chie ${ }^{1,2}$, Yong Chan Ahn ${ }^{34}$ \\ ${ }^{1}$ Department of Radiation Oncology, Seoul National University College of Medicine, Seoul, ${ }^{2}$ Institute of Radiation Medicine, Medical Research Center, \\ Seoul National University, Seoul, ${ }^{3}$ Department of Radiation Oncology, Samsung Medical Center, Sungkyunkwan University School of Medicine, Seoul, \\ ${ }^{4}$ Department of Medical Device Management and Research, SAIHST, Sungkyunkwan University, Seoul, Korea
}

Looking back the history of oncology, especially that of radiation therapy (RT), there have been several important pivotal technical innovations that provided the major breakthroughs. In most clinical settings, these have impacted and changed not only the concepts of RT, but also improved the consequent clinical outcomes following the advanced RT techniques. Particle beam therapy, which typically includes the irradiation of either proton beams or carbon ion beams, has currently become one of the major game-changers in radiation oncology field. The history of "particle physics" dates back to 1930s, when it was recognized as a distinct subject of modern physics, and the applicability of particle beams in treating the human patients was proved shortly after the World War II [1]. The clinical application of proton beam therapy using the dedicated equipment, however, began in 1990 at Loma Linda University Medical Center [2]. In Korea, there are currently two institutes operating the proton beam therapy equipment (National Cancer Center Korea and Samsung Medical Center), and two institutes are undergoing the installation process of carbon ion beam therapy facilities (Yonsei Cancer Center and Seoul National University Hospital). In addition, a few more institutes have committed or are considering to install their new particle beam therapy facilities.

Particle beam therapy, by virtue of the peculiar Bragg peak phenomenon and relative biologic effectiveness greater than 1 , is quite different from X-ray-based RT techniques. Sharing of accurate and balanced information regarding the basic and fundamental principle of particle beam therapy as well as the practical considerations in its optimal clinical applications in the real world situation seem to have been inadequate, even among the oncology specialists. Though 'something new' (and/or 'something expensive') are not always 'something better', there have been, however, numerous suggestive and illusive information, which has already been made widely public throughout the digital information channels. All medical practices need to be based on the "value" issue, which typically depends less on the cost exerted, but more on the outcomes on the patients' side [3-5]. There are still many issues that are prone to discussion, debate, and verification through the careful clinical research efforts.

In this context, we would sincerely wish that three special articles in the current issue of Cancer Research and Treatment (by Koom et al. [6], Kim et al. [7], and Park et al. [8], respectively) could provide the up-to-date and balanced insights on particle beam therapy, from the basic to the practical issues, to the interested readers who major in various oncology fields but may not be very familiar with this topic. Also we would wish these special articles to assist the Korean National Health Insurance Service in establishing the optimal reimbursement policy. In very reasonable senses, the current and future particle beam therapy providers in Korea are highly responsible to develop and update the optimal clinical indications, and thereby to provide the reliable clinical evidences on the benefits and the limitations of particle beam therapy to whoever may be interested in and concerned on these issues. By accomplishing these duties, the resources, to which the astronomical amount of cost and energy have already been invested, could be reasonably and optimally utilized. These efforts by the enthusiastic particle beam therapy providers, not only evidence-based but also evidence-making, will be continuously required, and should deserve the appreciation by our oncology societies accordingly.

\section{References}

1. Wilson RR. Radiological use of fast protons. Radiology. 1946; 47:487-91.

2. Slater JM, Archambeau JO, Miller DW, Notarus MI, Preston W, Slater JD. The proton treatment center at Loma Linda University Medical Center: rationale for and description of its development. Int J Radiat Oncol Biol Phys. 1992;22:383-9.

Correspondence: Yong Chan Ahn

Department of Radiation Oncology, Samsung Medical Center, Sungkyunkwan University School of Medicine, 81 Irwon-ro, Gangnam-gu, Seoul 06351, Korea Tel: 82-2-3410-2602 Fax: 82-2-6190-5332 E-mail: ahnyc@skku.edu

Published Online June 16, 2021 
3. Porter ME. What is value in health care? N Engl J Med. 2010;363:2477-81.

4. Hurst L, Mahtani K, Pluddemann A, Lewis S, Harvey K, Briggs A, et al. Defining value-based healthcare in the NHS [Internet]. Oxford: Centre for Evidence-Based Medicine, University of Oxford; 2019 [cited 2021 Jun 15]. Available from: https://www.cebm.net/ wp-content/uploads/2019/04/Defining-Value-based-healthcare-in-the-NHS_201904.pdf.

5. Teisberg E, Wallace S, O'Hara S. Defining and implementing value-based health care: a strategic framework. Acad Med. 2020;95:682-5. 6. Byun HK, Han MC, Yang K, Kim JS, Yoo GS, Koom WB, et al. Physical and biological characteristics of particle therapy for oncologists. Cancer Res Treat. 2021;53:611-20.

7. Kim KS, Wu HG. Who will benefit from charged-particle therapy? Cancer Res Treat. 2021;53:621-34.

8. Park JM, Kim JI, Wu HG. Technological advances in charged particle therapy. Cancer Res Treat. 2021;53:635-40. 